\title{
The prevalence of ESBL and AmpC $\beta$-lactamases in uropathogenic isolates of Escherichia coli in a tertiary care hospital in Southwest Iran
}

Mohammad Motamedifar ( $\sim$ motamedm@sums.ac.ir)

Shiraz University of Medical Sciences https://orcid.org/0000-0003-4993-4034

\section{Samane Mohebi}

Shiraz University of Medical Sciences

Mahtab Hadadi

Shiraz University of Medical Sciences

Hamideh Amirzadegan

Shiraz University of Medical Sciences

\section{Research note}

Keywords: AmpC $\beta$-lactamases, E. coli, ESBLs, uropathogenic Escherichia coli (UPEC)

Posted Date: January 10th, 2020

DOI: https://doi.org/10.21203/rs.2.20615/v1

License: (9) This work is licensed under a Creative Commons Attribution 4.0 International License.

Read Full License

Version of Record: A version of this preprint was published at Gene Reports on September 1st, 2020. See the published version at https://doi.org/10.1016/j.genrep.2020.100747. 


\section{Abstract}

Objectives: The spread of extended-spectrum $\beta$-lactamases (ESBLs) and AmpC $\beta$-lactamases (AmpC) in Escherichia coli ( E. coli) is an important public health concern and ESBL-producing bacteria are commonly reported in uropathogenic Escherichia coli (UPEC). The aim of this study is to determine the molecular detection of AmpC and ESBLs, among clinical E. coli isolated from inpatients who presented with symptomatic urinary tract infection (UTI) in Shiraz, the southwest of Iran.

Results: Among total 177 urinary isolates, the analysis showed that $46.3 \%$ of the isolates were ESBLs positive and that fifteen strains revealed the AmpC cluster genes. Among all ESBL-positive E. coli isolates, the CTX-M was the most prevalent ESBL type (68.2\%), and in AmpC-positive isolates, fifteen isolates (88.2\%) were positive for CITM cluster gene and two (11.7\%) were positive for DHAM cluster gene. ACCM, FOXM , EBCM and MOXM cluster genes were not found in this study. Our findings revealed that the prevalence of AmpC $\beta$-lactamases is rising in Iran, leading to failure in treatment. Therefore, the current study recommended that accurate and precise methods and guidelines should be designed for detection of antibiotic-resistant mechanisms because it is very important for the treatment and prevention of such isolates.

\section{Introduction}

Extended-spectrum $\beta$-lactamases (ESBLs) are the major cause of acquired drug resistance seen in gramnegative bacteria (GNB)(1). The ability of ESBL enzymes to hydrolyze most of $\beta$-lactam antibiotics is not active against cephamycins and carbapenems(2). Furthermore, production of AmpC $\beta$-lactamases is another mechanism that usually confers resistance to all $\beta$-lactams. Importantly, Amp $C$ is a major clinical concern since these enzymes play a significant role in conferring cephamycins hydrolyze and also are able to increase the minimum inhibitory concentration (MIC) value of carbapenems $(3,4)$. ESBLs and AmpC $\beta$-lactamases production can be chromosomal or plasmid-mediated and can be spread by horizontal transfer between gram-negative bacteria members $(5,6)$.

Urinary tract infection (UTI) caused by Escherichia coli (E. coli) is the most common infectious disease that accounts for a substantially increased morbidity, mortality, and health care costs(7). Co-production of ESBLs and AmpC $\beta$-lactamases occurred in some of the isolates pathogens; principally E. coli, is increasing and causes a broader spectrum of resistance $(8,9)$. In recent years, the prevalence of $\beta$ lactamase (bla) genes, such as blaSHV, blaTEM, blaCTX-M, and AmpC- $\beta$ - lactamase has been increasing in clinical isolates of E. coli obtained from patients with UTIs(10). It seems necessary to identify the AmpC b-lactamases producing bacteria in clinical isolates. To this end, we aim to identify the prevalence of ESBLs and also detect blaSHV, blaTEM, blaCTX-M and AmpC $\beta$-lactamase genes by using designed primers through PCR assay in clinical isolates of E. coli obtained from patients with UTIs from Shiraz, Iran.

\section{Materials And Methods}


One hundred seventy-seven nonconsecutive, nonduplicate clinical E. coli urine isolates were obtained from November 2017 to May 2018 from inpatients hospitalized at a tertiary-care teaching hospital (Namazi) in Shiraz, Iran. The isolates were from both male and female inpatients diagnosed with UTI who presented at different wards of Namazi Hospital such as ICU, surgery, internal, transplant. All samples were detected by IMVIC standard biochemical tests including indole, methyl red, Voges-Proskauer, and citrate. Confirmed E. coli isolates were kept frozen in tryptic soy broth (Merck Co., Germany) containing $20 \%$ glycerol (Merck KGaA, Germany) at $-70{ }^{\circ} \mathrm{C}$ for further usage in the tests. This study was approved by the Ethics Committee of Shiraz University of Medical Sciences Sciences. The informed consent was obtained from all the participants, and informed consent obtained was written.

Phenotypic Tests for Detection of ESBL

ESBL production was assessed using the CLSI recommendations for ESBL screening and phenotypic confirmation tests(11). The following antibiotics (Mast, UK) were used for detecting ESBLs: CAZ (30 $\mu \mathrm{g})$, CTX $(30 \mu \mathrm{g})$ alone as well as with $10 \mu \mathrm{g}$ clavulanic acid. Klebsiella pneumonia ATCC 700603 (ESBLs positive) and E. coli ATCC 25922 (ESBLs negative) were used as the control strains. The double-disc synergy test (DDST) was carried out for the phenotypic confirmation of ESBL production.

AmpC screening and confirmatory test

Screening for AmpC $\beta$-lactamase production was done by placing a cefoxitin disk (30 $\mu \mathrm{g}$; Mast Diagnostics Ltd., UK) on Mueller-Hinton agar(11). Isolates showing an inhibition zone diameter of < $18 \mathrm{~mm}$ were considered positive on the screening test and subjected to confirmatory phenotypic methods for detection of AmpC $(12,13)$. E. coli ATCC 25922 was used as the negative control in the AmpC disk test method.

\section{DNA Extraction and Molecular Detection of AmpC sand bla Genes}

Genomic DNA of positive screens for ESBLs and AmpC was extracted by extraction kit (Bioneer, Seoul, Korea), according to the manufacturer's instructions.

The detection of the six different families of plasmid-mediated AmpC B-lactamases including ACC, CIT, DHA, EBC, FOX, and MOX was done using the primers designed by Perez-P'erez and Hanson (14). The $\beta$ lactamase genes blaTEM, blaSHV, and blaCTX-M were detected in the ESBL-positive isolates by PCR using the specific oligonucleotide primers(15).

Statistical Analysis

Data management and analysis were performed using the Statistical Package for Social Sciences (SPSS; Version 22.0). Simple descriptive statistics were presented to analyze the outcome data. Chi-square test 
was used to compare between the resistant patterns of $\beta$-lactamase- and non- $\beta$-lactamase-producing isolates.

\section{Results}

\section{Distribution of the isolates from Clinical Specimens}

The recruited patients in our study were 105 females and 72 males with an age range of 1-85 years old. The recovered UPEC isolates from different wards were as follows: Intensive Care Unit or ICU $(n=118$, $67 \%)$, Internal wards $(n=37,21 \%)$, Surgery $(n=13,7 \%)$, and Transplantation $(n=9,5 \%)$. Moreover, the frequencies of cases in different wards were as follows: cystitis (ICU $=56$, Internal ward $=12$, Surgery $=5$, Transplantation $=7$ ), pyelonephritis $(\mathrm{ICU}=47$, Internal ward $=22$, Surgery $=8$, Transplantation $=2)$, and urosepsis $(I C U=15$, Internal ward $=3$, Surgery $=0$, Transplantation $=0)$.

\section{Prevalence of ESBLs and AmpC $\beta$-Lactamases}

Of the 177 E. coli isolates examined for $\beta$-lactamases, $46.3 \%$ (82) were found to be ESBL producers and $18.6 \%$ (33) were suspected as AmpC producer by disk agar diffusion (DAD) cefoxitin screening method. By confirmatory phenotypic methods for detection of AmpC, $54.4 \%(n=18)$ of the isolate were found to be AmpC $\beta$-lactamase producer. A total of $18(40 \%)$ isolates were AmpC-positive, and $17(94.4 \%)$ isolates were ESBL producers. ESBL producers were commonly recovered from different wards as follows: ICU (65.8\%), Internal ward (22\%), Surgery (7.3\%), and transplantation (4.9\%)), whereas AmpC $\beta$-lactamase producers were more frequent in ICUs (72.2\%), Internal ward (22.2\%), and transplantation department $(5.6 \%)$.

Among the ESBL-producing isolates, $68.2 \%(n=56), 25.6 \%(n=21)$ and $6.2 \%(n=5)$ were positive for $b / a_{\mathrm{CTX}-\mathrm{M}}, b / a_{\mathrm{TEM}}$ and $b / a_{\mathrm{SHV}}$, genes, respectively. Also, the prevalence of the $b / a_{\mathrm{CTX}-\mathrm{M}}, b / a_{\mathrm{TEM}}$ and $b / a_{\mathrm{SHV}}$ ESBL genes in the AmpC-producing isolates were found to be $61.1 \%(n=11), 27.7 \%(n=5)$ and $1.1 \%(n=$ 2), respectively. The multiplex PCR findings of $17 \mathrm{AmpC}$-positive, ESBL producers revealed that fifteen isolates (88.2\%) were positive for CITM cluster gene and two isolates $(11.7 \%)$ were positive for DHAM cluster gene (Figure 1). ACCM, FOXM, EBCM and MOXM cluster genes were not found in this study. The distribution of resistance genes profile bla $a_{\mathrm{CTX}}, b / a_{\mathrm{TEM}}$ and $b / a_{\mathrm{SHV}}$ and AmpC cluster genes in $\mathrm{ESBL}$ and AmpC-producing isolates are presented in Tables 1, 2.

\section{Discussion}

The emergence of ESBLs and AmpC producing Enterobacteriaceae presents a major diagnostic and therapeutic problem in the limit of infections (14). In this study, ESBL was detected in 82 (46.3\%) of the 177 isolates recovered from patients, and the bla ${ }_{\text {CTX-M }}(56 / 82$ isolates; $68.2 \%)$ was found more frequently than the bla $\mathrm{TEM}_{\mathrm{TEM}}(21 / 82$ isolates; $25.6 \%)$ gene in ESBL-producing E. coli isolates. 
Our results showed that the incidence of ESBL in E. coli owing to the spread of bla $\mathrm{C}_{\mathrm{CTX}-\mathrm{M}}$ and bla $\mathrm{a}_{\mathrm{TEM}}$ enzymes was consistent with previous research in Iran, Egypt, India, Iraq, Turkey and Saudi Arabia (1621). Meanwhile, higher prevalence of ESBL producing E. coli was observed in ICU. The rate of ESBLproducing E.coli in ICUs varies greatly in different parts of the globe ranging from $13.2 \%$ in France(22) to as high as $61 \%$ in India (18). The prevalence of ESBL producing E.coli among the ICUs in Iran ranges from $11.4 \%(23)$ to $70.6 \%(24)$, whereas the prevalence rate in our study was $65.8 \%$.

In Iran, AmpC prevalence has been reported in E. coli (3.3\% and 5.7\%)(25). The frequency rates of AmpCproducing E. coli isolates in Iran is not clear due to limited number of studies and problems identifying that laboratories have detected the resistance mechanisms. The present study demonstrated that from the $33(18.6 \%)$ cefoxitin resistant isolates about $(54.4 \%, 18 / 33)$ isolates were AmpC ß-lactamase producer. Also, according to the PCR assay results, we found the prevalence of AmpC genes in 35.3\% AmpC-producing isolates. The result was consistent with those of the studies carried by Saffar et al. (5) and Fam et al. (26) from Egypt where AmpC prevalence was $15.83 \%$ and $28.3 \%$, respectively. On the other hand, the base on the geographical location, the species studied, the period of study, the prevalence, and type of acquired plasmid AmpC detected can be different(27). For this reason, comparison of the actual incidence of AmpCs in the studies is difficult. According to the PCR assay results, we found two types of cluster genes (CITM and DHAM) in fifteen isolates, indicating the prevalence of AmpC $\beta$-lactamases in Iran. The study by Mansouri et al. (28) indicated that among 88 clinical isolates of E. coli, five (7\%) had AmpC genes that belong to DHAM, CITM, and EBCM cluster genes. It was noticed in this study that some of the E. coli isolates harbored more than one AmpC gene family that was reported in several studies(29). Several studies have shown that the high percentage of AmpC-harbouring isolates results in clinical treatment failures with broad-spectrum cephalosporins. Reducing the spread of plasmid-mediated AmpC resistance in hospitals requires the identification of the genes involved in order to control the movement of this resistance mechanism(14).

\section{Conclusion}

In many clinical centers, no attention is paid to the identification of AmpC $\beta$-lactamases based on phenotypic or genetic screening tests. The results of many studies emphasize the need to improve identification methods for ESBL and AmpC-producing organisms in hospitals where extended-spectrum cephalosporins are in wide use.

\section{Limitations}

The study focused on inpatients who presented with symptomatic UTI and included only one hospitals in Shiraz, thereby not allowing extrapolation of our results to other regions.

\section{Abbreviations}


UTI: urinary tract infections; E. coli: Escherichia coli; PCR: polymerase chain reactions; CLSI: Clinical and Laboratory Standard Institute; MIC: minimum inhibitory concentration; UPEC: uropathogenic Escherichia coli; ICU: Intensive Care Unit.

\section{Declarations}

\section{Acknowledgment}

The authors wish to thank Ms. M. Moradi for her invaluable assistance in editing this manuscript.

\section{Funding}

This study was supported by Shiraz University of Medical Sciences with grant No. 96-14629. This article was derived from the MSc thesis by Ms Hamideh Amirzadegan under the supervision of Dr. M Motamedifar.

\section{Author contributions}

All authors contributed to data analysis, drafting or revising the article, gave final approval of the version to be published, and agree to be accountable for all aspects of the work.

\section{Availability of data and materials}

All relevant data are included in the manuscript.

\section{Competing interests}

The authors declare that they have no competing interests.

\section{Ethics approval and consent to participate}

This project was approved by Shiraz University of Medical Sciences Ethics Committee, and all informed participants complemented a written consent.

\section{Consent to publish}

Not applicable.

\section{References}

1. Ruh E, Zakka J, Hoti K, Fekrat A, Guler E, Gazi U, et al. Extended-spectrum $\beta$-lactamase, plasmidmediated AmpC $\beta$-lactamase, fluoroquinolone resistance, and decreased susceptibility to carbapenems in Enterobacteriaceae: fecal carriage rates and associated risk factors in the community of Northern Cyprus. Antimicrob Resist Infect Control. 2019;8(1):98. 
2. Dortet L, Poirel L, Nordmann P. Rapid detection of extended-spectrum- $\beta$-lactamase-producing Enterobacteriaceae from urine samples by use of the ESBL NDP test. J Clin Microbiol.2014;52(10):3701-6.

3. van Hoek AH, Schouls L, van Santen MG, Florijn A, de Greeff SC, van Duijkeren E. Molecular characteristics of extended-spectrum cephalosporin-resistant Enterobacteriaceae from humans in the community. PloS one. 2015;10(6):e0129085.

4. Ghanavati R, Darban-Sarokhalil D, Navab-Moghadam F, Kazemian H, Irajian G, Razavi S. First report of coexistence of AmpC beta-lactamase genes in Klebsiella pneumoniae strains isolated from burn patients. Acta Microbiol Immunol Hung. 2017;64(4):455-62.

5. Saffar H, Niaraki NA, Tali AG, Baseri Z, Abdollahi A, Yalfani R. Prevalence of AmpC $\beta$-lactamase in Clinical Isolates of Escherichia coli, Klebsiella spp., and Proteus mirabilis in a Tertiary Hospital in Tehran, Iran. Jundishapur Journal of Microbiology. 2016;9(12): e39121.

6. Ibrahim ME, Abbas M, Al-Shahrai AM, Elamin BK. Phenotypic Characterization and Antibiotic Resistance Patterns of Extended-Spectrum $\beta$-Lactamase-and AmpC $\beta$-Lactamase-Producing GramNegative Bacteria in a Referral Hospital, Saudi Arabia. Can J Infect Dis Med Microbiol. 2019; 6054694.

7. Raeispour M, Ranjbar R. Antibiotic resistance, virulence factors and genotyping of Uropathogenic Escherichia coli strains. Antimicrob Resist Infect Control. 2018;7(1):118.

8. Deshpande LM, Jones RN, Fritsche TR, Sader HS. Occurrence of plasmidic AmpC type $\beta$-lactamasemediated resistance in Escherichia coli: report from the SENTRY Antimicrobial Surveillance Program (North America, 2004). Int J Antimicrob Agents. 2006;28(6):578-81.

9. Goossens H, Grabein B. Prevalence and antimicrobial susceptibility data for extended-spectrum $\beta$ lactamase-and AmpC-producing Enterobacteriaceae from the MYSTIC Program in Europe and the United States (1997-2004). Diagn Microbiol Infect Dis. 2005;53(4):257-64. 
10. Koshesh M, Mansouri S, Hashemizadeh Z, Kalantar-Neyestanaki D. Identification of extendedspectrum $\beta$-lactamase genes and ampc- $\beta$-lactamase in clinical isolates of escherichia coli recovered from patients with urinary tract infections in Kerman, Iran. Arch. Pediatr. Infect. Dis. 2017;5(2) ; e37968

11. CLSI. Performance Standards for Antimicrobial Susceptibility Testing; 28th Informational Supplement. CLSI Document M100. Wayne, PA: Clinical and Laboratory Standards Institute; 2018:2018.

12. Khari FIM, Karunakaran R, Rosli R, Tay ST. Genotypic and phenotypic detection of AmpC $\beta$ lactamases in Enterobacter spp. isolated from a teaching hospital in Malaysia. PloS one. 2016;11(3):e0150643.

13. Black JA, Moland ES, Thomson KS. AmpC disk test for detection of plasmid-mediated AmpC $\beta$ lactamases in Enterobacteriaceae lacking chromosomal AmpC $\beta$-lactamases. J Clin Microbiol. 2005;43(7):3110-3.

14. Pérez-Pérez FJ, Hanson ND. Detection of plasmid-mediated AmpC $\beta$-lactamase genes in clinical isolates by using multiplex PCR. J Clin Microbiol. 2002;40(6):2153-62.

15. Khurana S, Mathur P, Kapil A, Valsan C, Behera B. Molecular epidemiology of beta-lactamase producing nosocomial gram-negative pathogens from North and South Indian hospitals. J Med Microbiol. 2017;66(7):999-1004.

16. Haghighatpanah M, Nejad ASM, Mojtahedi A, Amirmozafari N, Zeighami H. Detection of extendedspectrum $\beta$-lactamase (ESBL) and plasmid-borne blaCTX-M and blaTEM genes among clinical strains of Escherichia coli isolated from patients in the north of Iran. J Glob Antimicrob Resist. 2016;7:110-3.

17. Abdallah H, Alnaiemi N, Reuland E, Wintermans B, Koek A, Abdelwahab A, et al. Fecal carriage of extended-spectrum $\beta$-lactamase-and carbapenemase-producing Enterobacteriaceae in Egyptian 
patients with community-onset gastrointestinal complaints: a hospital-based cross-sectional study. Antimicrob Resist Infect Control. 2017;6(1):62.

18. Singh N, Pattnaik D, Neogi DK, Jena J, Mallick B. Prevalence of ESBL in Escherichia Coli isolates among ICU patients in a tertiary care hospital. J Clin Diagn Res: JCDR. 2016;10(9):DC19 -DC22.

19. Polse RF, Yousif SY, Assafi MS. Prevalence and molecular characterization of extended spectrum beta-Lactamases-producing uropathogenic Escherichia coli isolated in Zakho, Iraq. Journal of Microbiology and Infectious Diseases. 2016;6(4):163-7.

20. Cicek AC, Saral A, Duzgun AO, Yasar E, Cizmeci Z, Balci PO, et al. Nationwide study of Escherichia coli producing extended-spectrum $\beta$-lactamases TEM, SHV and CTX-M in Turkey. J Antibiot. 2013;66(11):647.

21. Alqasim A, Abu Jaffal A, Alyousef AA. Prevalence of Multidrug Resistance and Extended-Spectrum $\beta$ Lactamase Carriage of Clinical Uropathogenic Escherichia coli Isolates in Riyadh, Saudi Arabia. Int J Microbiol.2018: 3026851.

22. Repessé X, Artiguenave M, Paktoris-Papine S, Espinasse F, Dinh A, Charron C, et al. Epidemiology of extended-spectrum beta-lactamase-producing Enterobacteriaceae in an intensive care unit with no single rooms. Ann Intensive Care. 2017;7(1):73.

23. Ghotaslou R, Sadeghi MR, Akhi MT, Hasani A, Asgharzadeh M. Prevalence and antimicrobial susceptibility patterns of ESBL, ampC and carbapenemase-producing enterobactericeae isolated from hospitalized patients in Azerbaijan, Iran. Iran J Pharm Res. 2018;17(Suppl):79-88.

24. Ashrafian F, Askari E, Kalamatizade E, Ghabouli-Shahroodi M, Naderi-Nasab M. The Frequency of Extended Spectrum Beta Lactamase (ESBL) in Escherichia coli and Klebsiella pneumoniae: A Report from Mashhad, Iran. Journal of Medical Bacteriology. 2013;2(1-2):12-9. 
25. Shayan S, Bokaeian M. Detection of ESBL- and AmpC-producing E. coli isolates from urinary tract infections. Adv Biomed Res. 2015;4:220.

26. Fam N, Gamal D, El Said M, Aboul-Fadl L, El Dabei E, El Attar S, et al. Detection of plasmid-mediated AmpC beta-lactamases in clinically significant bacterial isolates in a research institute hospital in Egypt. Life Sci J. 2013;10(2):2294-304.

27. Empel J, Baraniak A, Literacka E, Mrówka A, Fiett J, Sadowy E, et al. Molecular survey of $\beta$ lactamases conferring resistance to newer $\beta$-lactams in Enterobacteriaceae isolates from Polish hospitals. Antimicrob Agents Chemother. 2008;52(7):2449-54.

28. MANSOURI S, CHITSAZ M, HAJI HR, MIRZAEI M, GHEYNI M. Determination of resistance pattern of plasmid-mediated ampc $\beta$-lactamases producing isolate of Escherichia coli. DANESHVAR MEDICINE. 2009;16(80): 61-70.

29. Mohamudha PR, Harish BN, Parija SC. Molecular description of plasmid-mediated AmpC $\beta$ lactamases among nosocomial isolates of Escherichia coli \& Klebsiella pneumoniae from six different hospitals in India. Indian J Med Res. 2012;135(1):114-9.

\section{Tables}

Table 1. Distribution of resistance genes profile in ESBL-positive isolates 


\begin{tabular}{|l|l|l|}
\hline Genes & Frequency & Percent (\%) \\
\hline CTX-M & 17 & 20.7 \\
\hline CTX-M/ TEM/ CITM & 17 & 20.7 \\
\hline CTX-M/ TEM & 10 & 12.2 \\
\hline CTX-M/ CITM & 4 & 4.9 \\
\hline CTX-M/ SHV/ CITM & 4 & 4.9 \\
\hline TEM & 3 & 3.7 \\
\hline CTX-M/ CITM/ DHAM & 2 & 2.4 \\
\hline CTX-M/ SHV & 1 & 1.2 \\
\hline CTX-M/ TEM/ DHAM & 1 & 1.2 \\
\hline CITM & 1 & 1.2 \\
\hline No gene & 22 & 26.8 \\
\hline Total & 82 & 100.0 \\
\hline
\end{tabular}

Table 2. Distribution of resistance genes profile in AmpC-positive isolates

\begin{tabular}{|l|l|l|}
\hline Genes & Frequency & Percent (\%) \\
\hline CTX-M/ TEM/ CITM & 5 & 27.8 \\
\hline CTX-M/ CITM & 4 & 22.2 \\
\hline CTX-M/ SHV/ CITM & 2 & 11 \\
\hline CTX-M/ CITM/ DHAM & 2 & 11 \\
\hline CTX-M & 1 & 5.6 \\
\hline TEM & 1 & 5.6 \\
\hline CITM & 1 & 5.6 \\
\hline TEM/ CITM & 1 & 5.6 \\
\hline CTX-M/ TEM/ DHAM & 1 & 5.6 \\
\hline Total & 18 & 100 \\
\hline
\end{tabular}

Figures 


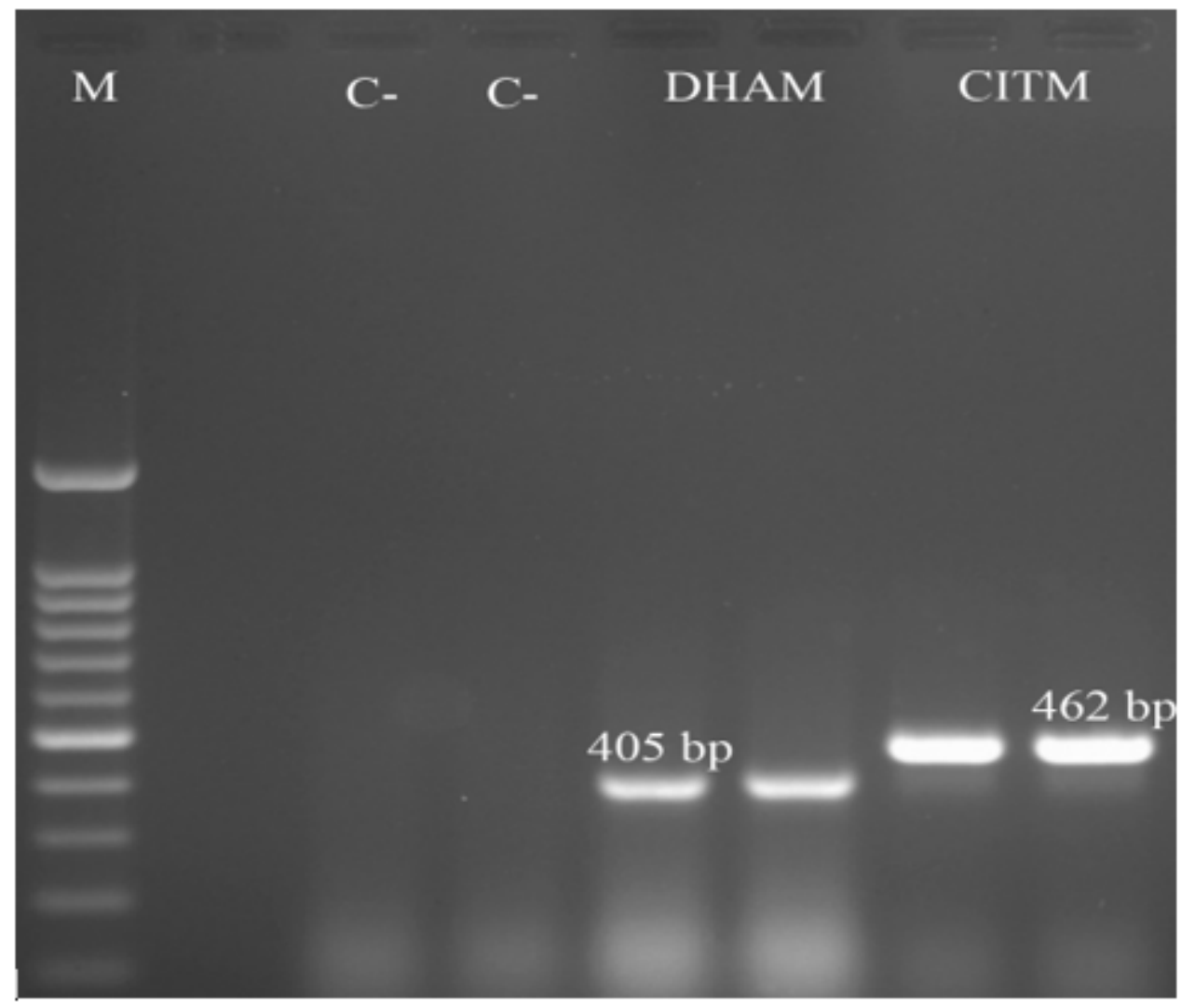

\section{Figure 1}

Representative image of PCR product electrophoresis; $1=$ DNA marker, $2=$ blank 3,4= negative control, $5,6=$ DHAM (405 bp) and 7,8 CITM (462 bp) 